\title{
REACTORES DISCONTINUOS SECUENCIALES: UNA TECNOLOGÍA VERSÁTIL EN EL TRATAMIENTO DE AGUAS RESIDUALES
}

\section{SEQUENTIAL BATCH REACTORS: A VERSATILE TECHNOLOGY FOR WASTEWATER TREATMENT}

\author{
Juan Fernando Muñoz Paredes', Mauricio Ramos Ramos²
}

Fecha de recepción: 13 de junio de 2013

Fecha de aprobación: 5 de mayo de 2014

Referencia: J.F. Muñoz Paredes, M. Ramos Ramos. (2014). Reactores discontinuos secuenciales: Una tecnología versátil en el tratamiento de aguas residuales. Ciencia e Ingeniería Neogranadina, 24 (1), pp 49 - 66.

\section{RESUMEN}

El interés en eliminar contaminantes de las aguas residuales se ha incrementado en los últimos años. Existe una constante búsqueda de nuevos y mejores diseños que permitan la implementación de sistemas de tratamiento confiables, de bajo costo y que ofrezcan mejores resultados. Esta tarea ha sido realmente un reto, debido a la generación de múltiples tipos de vertimientos líquidos, con diferentes características y composición y, en particular, al cumplimiento de estrictas y diferentes regulaciones que en los distintos países se establecen en materia de control ambiental. La investigación en el área de la ingeniería y las ciencias ambientales ha permitido el desarrollo de la tecnología de reactores discontinuos secuenciales (SBR, por sus siglas en inglés), como una variación del proceso de lodos activados convencional para la eliminación de materia orgánica y de nutrientes de las aguas residuales. El presente artículo de revisión describe la importancia de este tipo de sistemas, teniendo en cuenta las generalidades del tratamiento, la descripción técnica del sistema, los parámetros de operación más importantes, el mecanismo biológico del proceso de eliminación y las diferentes modificaciones al diseño original. Finalmente, se encontró que este tipo de sistema es de gran utilidad y ofrece muchas ventajas en la eliminación de nutrientes y materia orgánica, en comparación con los sistemas convencionales de tratamiento, además de que se puede aplicar para el tratamiento de gran cantidad y diversidad de aguas residuales tanto domésticas como industriales.

Palabras Clave: nutrientes, reactor, eliminación, tecnología SBR.

1. Ing. Químico, MSc. Ingeniería Ambiental, jfmunoz@umariana.edu.co, Docente Facultad de Posgrados y Relaciones Internacionales, Grupo de Investigación GIA. Universidad Mariana, Pasto, Colombia.

2. Ing. Químico, MSc. Ciencias de la Ingeniería, mauramos2000@gmail.com, Docente Facultad de Ingeniería, Maestría en Ingeniería Ambiental (En curso). Grupo de Investigación GIA. Universidad Mariana, Pasto, Colombia. 


\section{ABSTRACT}

Interest in eliminating sewage polluting agents has increased in recent years. There is a constant search for new and better designs that allow reliable, affordable, and more efficient treatment systems implementation. This has been a challenging task due to the different characteristics and composition of wastewaters that need to be treated and, especially, to the variety of regulations that different countries set up in terms of environmental control. Research on the fields of engineering and environmental sciences has granted the development of sequential batch reactors (SBR) technology as a variation of the common activated sludge processes in order to eliminate the organic matter and nutrients in sewage sludge. This review article describes the importance of these types of systems, taking into account the treatment generalities, such as the system technical description, the most important operation parameters, the biological mechanism of the elimination process, and the different changes to the original design. Finally, it was found that this kind of system, compared to the conventional ones, is highly useful and offers numerous advantages in the elimination of organic matter and nutrients, as well as in the treatment of large quantities of both domestic and industrial sewage.

Keywords: nutrients, reactor, elimination, SBR technology.

\section{INTRODUCCIÓN}

Es evidente que en la actualidad el crecimiento de la población mundial ha incrementado la cantidad y la diversidad de las aguas residuales, tanto en materia orgánica como en nutrientes, de tal forma que su disposición incontrolada causa deterioro en el ambiente, al propiciar el proceso denominado eutrofización [1-23]. La presencia de estos compuestos es esencial para el crecimiento de las plantas y de otros organismos, por lo que se denominan nutrientes [4-5-6].

El vertido de aguas residuales ricas en estos compuestos puede dar lugar a la eutrofización, entendida como un proceso complejo de fertilización de las aguas naturales con sustancias nutritivas, especialmente nitrógeno y fósforo en formas asimilables por la vegetación acuática. Este proceso origina un aumento de la población de algas, un incremento de la productividad en todos los niveles de la cadena alimentaria y un empeoramiento progresivo de las características fisicoquímicas del agua. Además existen otros impactos asociados a la eutrofización, como el descenso del oxígeno disuelto en los cuerpos de agua y la generación de un ambiente tóxico para los organismos acuáticos [7].

Por otro lado, los nutrientes son los factores que más importancia tienen en la eutrofización de las fuentes de agua. Para controlar este fenómeno se deben implementar las acciones tendientes a 
reducir la descarga de estos contaminantes, más aun teniendo en cuenta que se han incrementado los niveles de exigencia en la normatividad ambiental vigente en muchos países del mundo [8-9].

Teniendo en cuenta la problemática expuesta anteriormente, los reactores discontinuossecuenciales(SBR) constituyen una tecnología versátil en el tratamiento de las aguas residuales y continúan adquiriendo importancia en la eliminación de contaminantes, con respecto a su similar de lodos activados convencional.

SBR es el nombre dado al sistema de tratamiento de aguas residuales operado sobre la base de la tecnología de lodos activados, en una secuencia de ciclos de llenado y de vaciado. Éste incluye normalmente el proceso de eliminación biológica de nutrientes, en fases que pueden incluir el tratamiento anaerobio, aerobio, anóxico, o la combinación de ellos y en las que, finalmente, se incluye la sedimentación. Todas estas operaciones unitarias se desarrollan en un mismo reactor [10].

Este tipo de reactores constituyen una excelente oportunidad de innovación en el campo de tratamiento de aguas residuales, debido a la flexibilidad de operación y a su fácil automatización. Así mismo, en años recientes, los sistemas de reactores discontinuos secuenciales han demostrado buenos resultados en la eliminación biológica de nutrientes. Este sistema de tratamiento ofrece varias ventajas incluyendo mínimo requerimiento de espacio, facilidad de manejo y la posibilidad de realizar modificaciones en el tren de tratamiento [11].

El presente artículo de revisión es importante puesto que permite un mayor acercamiento a la tecnología de sistemas SBR, de tal manera que permite un mejor conocimiento de su funcionamiento y de los principales parámetros de operación. Igualmente, constituye un punto de referencia para investigadores que gustan de este tema de estudio, para la comunidad académica relacionada con el saber específico y para las entidades públicas y privadas que se interesan en el manejo y preservación del recurso hídrico.

\section{GENERALIDADES DE LA TECNOLOGÍA SBR}

Los SBR seconocen hace aproximadamente 100 años, desde que Arden y Lockett publicaron en 1914 trabajos realizados en reactores a escala piloto, en ese tiempo llamados reactores de llenado y vaciado. Estudios posteriores no tuvieron éxito hasta que en 1971, Irvine y David retomaron el desarrollo de estos reactores y en 1983 Sir Thomas Wardle publicó sus experiencias y así permitió que estudios similares se reanudaran ampliamente en varios países [12].

Un reactor discontinuo secuencial (SBR) se puede definir como un sistema de lodos activados, cuyo funcionamiento se basa en la secuencia de llenado y vaciado. Los procesos unitarios que intervienen son similares a los de un proceso 
convencional de lodos activados. Ambos sistemas intervienen la mezcla, reacción y sedimentación, pero entre ambos existe una diferencia importante, ya que, en las plantas convencionales, los procesos se llevan a cabo simultáneamente en tanques separados, mientras que en un sistema SBR los procesos tienen lugar en el mismo tanque [12].

En contraste con el sistema continuo, el agua se introduce al reactor en un tiempo establecido previamente, la degradación de la materia orgánica y la sedimentación se realizan en el mismo tanque. Las etapas del proceso son secuenciales y se repiten periódicamente, además de que se emplea aireación (proceso aerobio) o no (proceso anaerobio), para conseguir la degradación de la materia orgánica y la eliminación de nitrógeno y de fósforo [12].

Los sistemas de reactores discontinuos tienen en común 4 etapas, las cuales se llevan a cabo en secuencia: etapa de llenado, para la adición de sustrato al reactor; etapa de reacción, en la cual el reactor se somete o no a aireación; dependiendo de las necesidades del tratamiento, etapa de sedimentación que permite la separación de sólidos para lograr un sobrenadante clarificado como efluente; y etapa de vaciado, cuyo propósito es la extracción del agua clarificada del reactor [13].

Hay que mencionar además, que los SBR fueron usados originalmente para la eliminación de demanda química de oxígeno (DQO) y fosfatos de las aguas residuales. La regulación en las descargas resultó en la modificación de estos sistemas para lograr la nitrificación y desnitrificación, junto con la eliminación de DQO y de fosfatos [14]. Algunos autores [15-16] sostienen que la eliminación biológica de nutrientes y de materia orgánica se obtiene gracias a la combinación de ciclos de reacción aerobios y anóxicos, dependiendo de las necesidades del tratamiento.

El sistema SBR ha sido utilizado con éxito para el tratamiento de aguas residuales provenientes de la industria química, para el tratamiento de estiércol de cerdo, aguas residuales salinas y suelos contaminados entre otros efluentes [15-17-18-19-20]. Además de permitir el manejo de un amplio espectro de compuestos orgánicos, y con la incorporación de etapas anaeróbicas/ anóxicas, se puede lograr la remoción conjunta de materia orgánica (MO), nitrógeno (N) y fósforo (P) [21].

Con la introducción de la fase anaerobia se produce la liberación de fósforo por parte de los microorganismos; en la fase aerobia tiene lugar la nitrificación, consumo de oxígeno y de fósforo; mientras que la desnitrificación ocurre en la siguiente fase anóxica. Debido a que en la operación de un SBR el sustrato orgánico soluble es consumido por los microorganismos en la fase aerobia, se deben efectuar mediciones para asegurar suficiente sustrato orgánico soluble para la desnitrificación [13].

La intensidad en la aireación durante la fase aerobia en este tipo de reactores influye en el desempeño del proceso global. Para optimizar el desempeño y mejorar el 
control del proceso se deben implementar estrategias de control y realizar mediciones de parámetros en tiempo real. Algunos de los más utilizados son el potencial de oxidoreducción (ORP, por sus siglas en inglés), $\mathrm{pH}$ y el oxígeno disuelto (OD) [22-23].

Muchos de estos sistemas se construyeron en Australia, Estados Unidos y Japón. En países como Alemania y Japón se han realizado avances significativos en sistemas que emplean este tipo de reactores, y existe gran cantidad de información sobre técnicas de diseño y parámetros de operación para el tratamiento de aguas residuales domésticas e industriales [24]. En los últimos años se ha evolucionado en el tratamiento con reactores secuenciales discontinuos de biopelícula, los cuales tienen la ventaja de remover nitrógeno y fósforo en un solo reactor [25-26].

En cuanto a los reactores de biopelícula, éstos permiten que la presencia de una zona anóxica facilite la nitrificación y la desnitrificación simultánea durante la fase aerobia. En este caso, la nitrificación ocurre en la superficie de la biopelícula y la desnitrificación ocurre en las capas internas debido al gradiente de oxígeno [27]. Por otro lado, estos reactores ofrecen ventajas como ahorro de espacio y energía, mayor concentración de biomasa, operación flexible, menor sensibilidad a la toxicidad, baja producción de lodo y cargas volumétricas altas [28]. Además, en estos sistemas la biomasa adherida al medio de soporte es la que gobierna el proceso de tratamiento. Sin embargo, cuando se incrementa el espesor de la biopelícula pueden aparecer regiones aerobias y anaerobias, permitiendo la remoción simultánea de DBO (Demanda bioquímica de oxígeno) y de nitrógeno [29]

\section{OPERACIÓN DE SBR}

Los reactores SBR fundamentan su funcionamiento en un sistema de llenado y vaciado. Todos los procesos se llevan a cabo en un solo reactor y siguen una secuencia de llenado, reacción, sedimentación y vaciado. Con fundamento en lo planteado por Broch [30], la configuración del ciclo depende de las características del agua residual y los requisitos legales a cumplir, y sigue fundamentalmente las siguientes etapas:

Etapa de llenado: esta etapa puede ser estática, mezclada o aireada, dependiendo de los objetivos que se tengan previstos para el tratamiento del agua residual. En el Ilenado estático resulta una entrada mínima de energía y una concentración alta de sustrato al final de la misma. Puede presentarse desnitrificación con la presencia de nitratos y generar condiciones propicias para la remoción del fósforo. Con respecto al Ilenado aireado, se genera al comienzo de las reacciones aerobias y mantiene bajas concentraciones de sustrato, situación importante cuando existen elementos tóxicos en el agua residual. En cualquier caso, la fase de alimentación puede ser simple o múltiple dependiendo de los objetivos del tratamiento [31].

Etapa de reacción: generalmente se proveen condiciones de mezcla, en las que se permite el consumo de sustrato en condiciones ambientales controladas que 
pueden ser aerobias, anaerobias o anóxicas, dependiendo de lasecuencia de tratamiento elegida. En las investigaciones asociadas a los procesos SBR se explica que en la fase de reacción anaerobia esencialmente se propicia la liberación del fósforo en forma de ortofosfatos; en la aerobia se llevan a cabo la oxidación de la materia orgánica y la nitrificación; y en condiciones anóxicas se presenta la desnitrificación y asimilación de fósforo [13].

Etapa de sedimentación: la obtención del lodo es otro paso importante en la operación de este tipo de reactores, que afecta en gran medida el rendimiento [32]. Su objetivo es la regulación de la concentración de sólidos en el lodo en el reactor. Este lodo podría obtenerse al final de la fase de reacción o durante la fase de sedimentación. Los sólidos se dejan separar del líquido en condiciones de quietud, lo que resulta en un sobrenadante clarificado que puede ser descargado como efluente. El tiempo de asentamiento puede durar entre 0,5 y 1,5 h, y previene que el manto de sólidos flote debido a la acumulación de gas [33].

Etapa de vaciado: el sobrenadante clarificado se descarga del reactor como efluente, mediante un mecanismo que debe ser diseñado y operado de manera que se evite que el material flotante sea descargado. El exceso de lodo activado residual también se remueve, empleando un tiempo que puede variar desde un 5 a un $30 \%$ del tiempo total.

El proceso llevado a cabo en un SBR puede ofrecer muchas características ventajosas para aplicaciones en investigación: el control de las condiciones de funcionamiento es más fiable, preciso y versátil, la recolección de datos por medio de sensores de toma de muestras y en línea se hace más fácil y los resultados son más representativos [34].

De igual manera, la operación en un mismo tanque permite el ahorro en costos de capital y cuenta con ventajas tales como la posibilidad de ajustar y cambiar la duración de las diferentes etapas de tratamiento, control final de cada reacción biológica, así como también de la calidad del efluente [35-36].

El resurgimiento del interés en el estudio de los SBR se limitó inicialmente a aplicaciones de tratamiento de los pequeños generadores de aguas residuales. Sin embargo, la necesidad de una mayor eficiencia en la eliminación de nutrientes, bajo límites cada vez más estrictos se ha traducido en la adopción de la tecnología SBR en instalaciones tan grandes como las que tratan 660 L/s [37].

\section{ELIMINACIÓN DE NUTRIENTES}

\subsection{ELIMINACIÓN DE NITRÓGENO}

La remoción de nitrógeno básicamente implica la conversión aerobia de amonio a nitrato (nitrificación) y la conversión anóxica de nitrato en gas nitrógeno (desnitrificación). La nitrificación se realiza por bacterias nitrificantes autotróficas, que son aerobias obligadas (nitrosomonas y nitrobacter) [33].

La reacción de nitrificación es exotérmica y en una primera fase el amonio se convierte en nitrito mediante la siguiente reacción [38]. 


$$
\mathrm{NH}_{4}^{+}+\frac{1}{2} \mathrm{O}_{2} \rightarrow \mathrm{NO}_{2}^{-}+2 \mathrm{H}^{+}+\mathrm{H}_{2} \mathrm{O} \quad\left(\Delta \mathrm{G}=-66 \frac{\mathrm{kcal}}{\mathrm{mol}}=-277 \frac{\mathrm{kJ}}{\mathrm{mol}}\right)
$$

Posteriormente el nitrito, que es un producto intermedio de la nitrificación y que

$$
\mathrm{NO}_{\overline{2}}+\frac{1}{2} \mathrm{O}_{2} \rightarrow \mathrm{NO}_{3}^{-} \quad\left(\Delta \mathrm{G}=-17 \frac{\mathrm{kcal}}{\mathrm{mol}}=-71,4 \frac{\mathrm{kJ}}{\mathrm{mol}}\right)
$$

permanece por corto tiempo, se convierte en nitrato de la siguiente forma [38]:
La desnitrificación es un proceso por el cual el nitrógeno en forma de nitrato es reducido a nitrógeno gas $\left(\mathrm{N}_{2}\right)$ que se libera a la atmósfera, por intermedio de bacterias heterótrofas aerobias facultativas que en condiciones anóxicas son capaces de utilizar los nitratos como aceptores de electrones, en lugar del oxígeno disuelto [33].

Este proceso se realiza en varias etapas: el nitrato $\left(\mathrm{NO}_{3}{ }^{-}\right)$se convierte en nitrito $\left(\mathrm{NO}_{2}^{-}\right)$, óxido nítrico (NO), oxido nitroso
$\left(\mathrm{N}_{2} \mathrm{O}\right)$ y finalmente en nitrógeno molecular $\left(\mathrm{N}_{2}\right)$, mediante la acción de diversos géneros de bacterias como Pseudomonas y Alcaligenes principalmente, pero también por géneros como Azospirillum, Rhizobium, Rhodopseudomonas y Propionibacterium en determinadas condiciones [39].

La desnitrificación se puede presentar al mismo tiempo que se oxida la materia orgánica, como se muestra en la siguiente reacción:

$$
\mathrm{C}_{6} \mathrm{H}_{12} \mathrm{O}_{6}+4 \mathrm{NO}_{3}^{-} \rightarrow 6 \mathrm{CO}_{2}+6 \mathrm{H}_{2} \mathrm{O}+2 \mathrm{~N}_{2}
$$

La eliminación de nitrógeno en un sistema SBR se puede lograr por la alternancia de periodos aerobios y períodos de anoxia durante la reacción. En las diferentes secuencias de tratamiento de las aguas residuales en un sistema SBR, la inclusión del periodo anóxico inmediatamente después del período de aireación mejora la eficiencia de remoción del nitrógeno por desnitrificación [40].

También hay que señalar que las bacterias nitrificantes consumen oxígeno disuelto durante este proceso y producen a la vez una disminución de la alcalinidad del agua residual de $7,07 \mathrm{mg} \mathrm{CaCO}_{3} / \mathrm{mg}$ $\mathrm{NH}_{4}{ }^{+}-\mathrm{N}$ oxidado, mientras que el proceso de desnitrificación aporta alcalinidad al agua en una proporción próxima a 3,57 $\mathrm{mg} \mathrm{CaCO} 3 / \mathrm{mg} \mathrm{NO}_{3}{ }^{-}-\mathrm{N}$ reducido, lo que permite compensar en parte el consumo experimentado durante la nitrificación [41].

\subsection{ELIMINACIÓN DE FÓSFORO}

La eliminación del fósforo (P) es importante en el control de la eutrofización debido a que es un nutriente limitante en la mayor parte 
de los sistemas de agua dulce y conduce a cambios significativos en la calidad del agua. Este proceso de eliminación por medio de un proceso biológico se conoce como la eliminación biológica de fósforo mejorada (EBPR, por sus siglas en inglés) [42].

El proceso EBPR consiste en la incorporación del $P$ presente en el afluente en la biomasa celular, que posteriormente se retira del sistema como resultado de la purga de lodo residual. La eficiencia de eliminación de fósforo en este sistema puede alcanzar hasta el 90 $\%$, mientras que en sistemas convencionales de lodos activados la eficiencia máxima alcanzada es sólo del 10 al 20 \% [33].

Entre los factores más importantes en la eliminación de fósforo están la composición de las aguas residuales, la relación influente DQO:N:P, los cationes presentes en las aguas residuales, la temperatura, el tiempo de retención de sólidos (TRS), la duración del ciclo del SBR y el tipo de inóculo. También es muy importante el control de las fases anaerobias y aerobias [16].

Los organismos encargados de la eliminación de fosforo son los organismos acumuladores de fósforo (PAO, por sus siglas en inglés), que requieren dos condiciones diferentes, aerobias y anaerobias, a fin de favorecer la absorción de P. La población biológica en la eliminación de fósforo comprende al menos dos grupos: un grupo que utiliza oxígeno o nitrato como aceptor de electrones (Denitrifying PAO, por sus siglas en inglés), y otro que utiliza sólo oxígeno (aeróbic PAO, por sus siglas en inglés) [43].
La eliminación biológica mejorada EBPR tiene tres características principales: la absorción y almacenamiento anaerobio de materia orgánica, la liberación de fosfato anaerobio y la asimilación aerobia de fosfato muy por encima de las necesidades de crecimiento de las células [44]. No obstante hay que tener en cuenta que los organismos acumuladores de glucógeno (GAO, por sus siglas en inglés) compiten por sustrato fácilmente biodegradable con los PAO, bajo condiciones anaerobias sin asimilar ni liberar P [45-46].

Las principales ventajas de la EBPR son la reducción del costo de productos químicos y una menor producción de lodos en comparación con la precipitación química.

\subsection{ELIMINACIÓN SIMULTANEA DE NITRÓGENO Y FÓSFORO}

En un sistema SBR, los procesos convencionales anaerobio - aerobio que incorporan una zona anóxica para desnitrificación han sido aplicados para la eliminación de Ny Pen plantas detratamiento de aguas residuales a gran escala, y han mostrado un aumento de la relación de fosfato anóxico absorbido a fosfato aeróbico consumido del 11 al $64 \%$ [47], por lo tanto es posible lograr la nitrificación, desnitrificación y eliminación de fósforo en un solo reactor usando la tecnología SBR. El nitrógeno puede ser eliminado como gas nitrógeno $\left(\mathrm{N}_{2}\right)$ por el accionar combinado de autótrofos en la nitrificación bajo condiciones aerobias y de heterótrofos en la desnitrificación bajo condiciones anaerobias, mientras el fósforo sólo puede ser eliminado por su absorción en 
la biomasa que puede ser descargada desde el sistema como un lodo excedente [36].

El fósforo se libera en condiciones anaerobias, a través del metabolismo de los organismos acumuladores de fósforo (PAO) y existe extra asimilación del mismo en condiciones aerobias. Es por esto que en los últimos estudios se han combinado diferentes secuencias y tiempos de duración de las fases anaerobias, aerobias y anóxicas, precisamente para lograr las mayores eficiencias de eliminación simultánea de C, N y $P$ presentes en las aguas residuales [48].

La desnitrificación puede también llevarse a cabo por los organismos PAO desnitrificantes (DPAOs, por sus siglas en inglés) en los sistemas EBPR anaeróbicoanóxicos, al permitir la reducción simultánea nitrato/nitrito y la absorción de fósforo usando la misma DQO. Se encontró además que los DPAOs realizan exactamente los mismos procesos que los PAO, excepto que utilizan nitrato o nitrito como aceptor de electrones en lugar de oxígeno [49].

En contraste, en los sistemas convencionales la eliminación biológica de $\mathrm{N}$ y de $\mathrm{P}$ requiere de DQO, que es a menudo el sustrato limitante dependiendo del tipo de agua residual en tratamiento. La desnitrificación compite con la EBPR, desde que las bacterias desnitrificantes consuman una porción del sustrato antes de que este pueda ser utilizado por los organismos biológicos removedores de fósforo. En otras palabras, la transferencia de nitrato en la fase anaerobia inhibe la liberación de fosfato [11]. Con el propósito de contribuir a la solución de este problema, una serie de cambios se han introducido en los sistemas SBR, como el llenado del reactor con medios de soporte o medios granulares aerobios en lugar de lodos activados [50].

La eficiencia de la absorción de nutrientes se ve afectada por variaciones en la composición del afluente. No obstante, se hanadelantado investigacionesimportantes sobre tratamiento de aguas residuales para un gran espectro de efluentes, que van desde la aguas residuales domésticas hasta diferentes efluentes industriales, para la eliminación de materia orgánica, $N$ y $P$. Los SBR también han demostrado buenos resultados en el tratamiento de diferentes tipos de lixiviados provenientes de rellenos sanitarios en diversas partes del mundo $[51-56,40]$.

Por otra parte, la eliminación de N y P puede ser monitoreada empleando los valores de $\mathrm{pH}$ y del potencial de oxido-reducción (ORP, por sus siglas en inglés) como parámetros de control [11]. La eliminación simultánea de nitrógeno, carbono y fósforo en un SBR se hace posible por la alternancia de las fases aerobias y anaerobias, lo que requiere que el orden y la duración de las diferentes etapas de reacción sean cuidadosamente optimizadas [15].

Así mismo, el monitoreo del pH, ORP y la tasa de consumo de oxígeno (TCO) han hecho posible distinguir claramente algunos puntos con características diferentes, tales como los tiempos de terminación de la nitrificación, la oxidación del carbono, y la desnitrificación endógena y exógena. 
La identificación y el control de estos tres parámetros conducen a un incremento en la eficiencia de remoción de carbono y nutrientes y a una buena adaptación de variación de carga.

\section{EXPERIENCIAS EN EL TRATAMIENTO DE AGUAS RESIDUALES CON SBR}

Los reactores SBR se han utilizado para el tratamiento de diversos tipos de vertimientos, entre los que se encuentran aguas residuales domésticas, aguas residuales industriales, aguas sintéticas, lixiviados, entre otros. También se han empleado diversas configuraciones $y$ secuencias, dependiendo de la calidad del efluente a tratar y de las necesidades de cada tipo de tratamiento. Así, por ejemplo, en la actualidad se utiliza el sistema convencional de sustrato suspendido y también el sistema de crecimiento adherido.

El tipo de sistema que trabaja con crecimiento adherido se emplea con frecuencia en procesos de lodos activados modificados para mejorar la remoción de compuestos orgánicos y promover las tasas de nitrificación. Con el empleo de la tecnología de crecimiento microbiano adherido se han llevado a cabo innumerables investigaciones, que han probado las bondades de este tratamiento solo o en combinación con el crecimiento suspendido típico de lodos activados [32].
En un proceso de biopelícula maduro, la concentración de biomasa en el medio es mucho mayor que en la suspensión líquida, lo cual es un indicativo de que la eficiencia del proceso de tratamiento es dirigida por la biopelícula. Sin embargo, el aumento del espesor de la biopelícula hace que coexistan regiones aerobias y anaerobias las cuales permiten la remoción simultánea de DBO y de nitrógeno [29].

La combinación de lechos adheridos y suspendidos ofrece mayor versatilidad frente a cambios súbitos de las condiciones de trabajo de los reactores en cuanto a carga orgánica e hidráulica y las relaciones entre materia orgánica, nitrógeno y fósforo, entre otros.

Una modificación del proceso SBR es el proceso anammox, el cual permite la conversión autotrófica del amonio a nitrógeno gas mediante condiciones anaerobias. Esta modificación presenta algunas ventajas como bajo requerimiento de energía y no necesita fuentes de carbono adicionales. Sin embargo, las bacterias que siguen este proceso son sensibles a diversos factores como la concentración de sustrato y temperatura entre otras [57].

A continuación se presenta un resumen de algunos estudios representativos, que han empleado SBR con diferentes configuraciones y con diferentes tipos de efluentes. 
Tabla 1. Experiencias en el tratamiento de $A R$ con SBR.

\begin{tabular}{|c|c|c|c|}
\hline $\begin{array}{c}\text { Tipo de } \\
\text { Reactor/proceso }\end{array}$ & $\begin{array}{l}\text { Tipo de AR o } \\
\text { efluente }\end{array}$ & Eficiencia & Referencia \\
\hline $\begin{array}{l}\text { Convencional } \\
\text { aerobio - anóxico }\end{array}$ & AR sintética & Eliminación del $97 \%$ en DBO & [12] \\
\hline $\begin{array}{l}\text { Convencional } \\
\text { anóxico-aerobio }\end{array}$ & AR sintética & $\begin{array}{c}\text { Eliminación del } 96 \% \text { en DBO } \\
40 \% \text { en NT, } 40 \% \text { en PT }\end{array}$ & [58] \\
\hline $\begin{array}{c}\text { Convencional } \\
\text { anaerobio-anóxico- } \\
\text { aerobio }\end{array}$ & AR sintética & $\begin{array}{c}\text { Eliminación } 70 \% \text { en PT y del } 90 \% \text { en } \\
\text { NT }\end{array}$ & [11] \\
\hline $\begin{array}{c}\text { Convencional } \\
\text { Anaerobio-aerobio- } \\
\text { anóxico }\end{array}$ & AR doméstica & $\begin{array}{c}\text { Eliminación del } 92 \% \text { en DBO, } 52 \% \\
\text { NKT y } 67 \% \text { PT }\end{array}$ & [13] \\
\hline $\begin{array}{l}\text { Convencional } \\
\text { Aerobio }\end{array}$ & AR sintética & Eliminación de $90 \%$ en PT & [59] \\
\hline $\begin{array}{c}\text { Biopelícula } \\
\text { Anaerobio-aerobio }\end{array}$ & AR sintética & $\begin{array}{c}\text { Eliminación } 97 \% \text { en PT y del } 73 \% \text { en } \\
\text { NT }\end{array}$ & {$[60]$} \\
\hline $\begin{array}{l}\text { Biopelícula } \\
\text { Aerobio-anaerobio }\end{array}$ & AR sintética & $\begin{array}{c}\text { Eliminación del } 90 \% \text { en } \mathrm{N}-\mathrm{NH}_{3}, 87 \% \text { en } \\
\text { PT y } 95 \% \text { en DQO. }\end{array}$ & [61] \\
\hline $\begin{array}{c}\text { Biopelícula } \\
\text { Anaerobio-aerobio }\end{array}$ & AR sintética & $\begin{array}{c}\text { Eliminación del } 90 \% \text { en PT y } 57 \% \text { en } \\
\text { NT }\end{array}$ & [6] \\
\hline $\begin{array}{c}\text { Biopelícula } \\
\text { Aerobio }\end{array}$ & AR industria láctea & $\begin{array}{c}92 \% \text { en DBO, } 94 \% \text { en DQO y } 73 \% \text { en } \\
\text { NT }\end{array}$ & [62] \\
\hline $\begin{array}{c}\text { Biopelícula } \\
\text { Anaerobio- } \\
\text { anóxico-aerobio }\end{array}$ & AR sintética & $\begin{array}{c}\text { Eliminación del } 77 \% \text { en NT y del } 33 \% \\
\text { en PT }\end{array}$ & [63] \\
\hline $\begin{array}{c}\text { Biopelícula } \\
\text { Aerobio-anaerobio }\end{array}$ & AR sintética & $\begin{array}{c}\text { Eliminación } 98 \% \text { en DQO, } 60 \% \text { en NT } \\
\text { y } 80 \text { en PT }\end{array}$ & [7] \\
\hline $\begin{array}{l}\text { Convencional } \\
\text { Aerobio-anaerobio }\end{array}$ & Lixiviado maduro & Eliminación del $90 \%$ en NT & [51] \\
\hline $\begin{array}{l}\text { Convencional } \\
\text { Aerobio }\end{array}$ & Lixiviado medio & $\begin{array}{l}\text { Eliminación del } 84 \% \text { en DQO, 44\% en } \\
\qquad-\mathrm{NH}_{4}^{+}\end{array}$ & [52] \\
\hline $\begin{array}{c}\text { Convencional } \\
\text { Anaerobio-aerobio }\end{array}$ & $\begin{array}{l}\text { Lixiviado } \\
\text { joven }\end{array}$ & $\begin{array}{l}\text { Eliminación del } 62 \% \text { en } \mathrm{DQO}, 31 \% \text { en } \\
\qquad \mathrm{N}-\mathrm{NH}_{4} \text { y } 19 \% \text { en } \mathrm{P}_{-} \mathrm{PO}_{4}^{-}\end{array}$ & [53] \\
\hline $\begin{array}{l}\text { Convencional } \\
\text { Aerobio }\end{array}$ & $\begin{array}{l}\text { Lixiviado } \\
\text { joven }\end{array}$ & Eliminación del 95\% en NT & {$[54]$} \\
\hline $\begin{array}{l}\text { Convencional } \\
\text { Aerobio-anaerobio }\end{array}$ & $\begin{array}{l}\text { Lixiviado } \\
\text { maduro }\end{array}$ & $\begin{array}{c}\text { Eliminación del } 98 \% \text { en DQO, } 80 \% \text { en } \\
\text { NKT }\end{array}$ & [55] \\
\hline $\begin{array}{l}\text { Convencional } \\
\text { Aerobio-anaerobio }\end{array}$ & $\begin{array}{l}\text { Lixiviado } \\
\text { maduro }\end{array}$ & $\begin{array}{c}\text { Eliminación del } 96 \% \text { en } \mathrm{N}-\mathrm{NH}_{4}{ }^{+} \text {y } 84 \% \\
\text { en DQO. }\end{array}$ & {$[56]$} \\
\hline
\end{tabular}


UNIVERSIDAD MILITAR NUEVA GRANADA - CIENCIA E INGENIERÍA NEOGRANADINA

\begin{tabular}{|c|c|c|c|}
\hline Convencional & Industria textil & $\begin{array}{c}\text { Eliminación del } 64 \% \text { en DBO, } 50 \% \text { en } \\
\text { NKT }\end{array}$ & [64] \\
\hline $\begin{array}{l}\text { Convencional } \\
\text { Aerobio }\end{array}$ & $\begin{array}{c}\text { Sintética } \\
\text { Industria } \\
\text { petroquímica } \\
\end{array}$ & $\begin{array}{c}\text { Eliminación del } 70 \% \text { en DQO, } 83 \% \text { en } \\
\qquad \mathrm{Hg} \text { y } 97 \% \text { en } \mathrm{Cd}\end{array}$ & [65] \\
\hline $\begin{array}{l}\text { Convencional } \\
\text { Aerobio }\end{array}$ & $\begin{array}{c}\text { Semiconductores } \\
\text { eléctricos }\end{array}$ & Eliminación del 95\% en DQO & [66] \\
\hline Convencional & $\begin{array}{c}\text { Acuacultura } \\
\text { Estiércol } \\
\text { Industria Alimentos } \\
\text { Industria química }\end{array}$ & $\begin{array}{c}80 \% \text { en DQO, } 60 \% \text { NT, } 85 \% \text { PT } \\
12 \% \text { DQO, } 20 \% \text { NT, 55\% PT } \\
70 \% \text { DQO, } 45 \% \text { NT, } 45 \% \text { PT } \\
48 \% \text { DQO, } 45 \% \text { NT, } 20 \% \text { PT }\end{array}$ & [67] \\
\hline $\begin{array}{l}\text { Convencional } \\
\text { Aerobio }\end{array}$ & Industria de Coque & $\begin{array}{c}\text { Eliminación de } 90 \% \text { en } \mathrm{N}^{-\mathrm{NH}_{4}}{ }^{+}, 69 \% \\
\text { en DQO }\end{array}$ & [68] \\
\hline
\end{tabular}

\section{CONCLUSIONES}

Si bien la eutrofización se produce en forma natural, normalmente está asociada a fuentes antrópicas, que generan un cambio acelerado de un estado trófico a un nivel superior debido a la adición de nutrientes a los cuerpos de agua, representados principalmente por compuestos de nitrógeno y fósforo.

La tecnología SBR es importante en el tratamiento de aguas residuales domésticas e industriales y ha adquirido gran importancia en los últimos años debido a la facilidad de operación y a la posibilidad de remover en una sola unidad materia orgánica y nutrientes. Además, ofrece otras ventajas en su aplicación y operación, como la precisión y versatilidad en el control de las condiciones de funcionamiento, la facilidad para la recolección de datos representativos por medio de sensores de toma de muestras y en línea, el ahorro en costos de capital en aspectos constructivos y de operación, la posibilidad de ajustar y cambiar la duración de las diferentes etapas de tratamiento, y el control final de cada reacción biológica y de la calidad del efluente.

La remoción simultánea de nitrógeno, carbono y fósforo en reactores SBR se hace posible al alternar las fases aerobias, anaerobias y anóxicas, lo que requiere de un adecuado estudio y planificación por parte de los operadores de este tipo de sistemas.

El sistema SBR con biopelícula ofrece muchas ventajas en la eliminación de contaminantes de las aguas residuales domésticas, incluyendo la eliminación de nitrógeno y fósforo. Este sistema posee características simples, estables y confiables, en comparación con el sistema de lodos activados convencional y facilita la implementación de sistemas compactos que ahorran espacio, evitan problemas de lodo voluminoso, son de fácil operación, de alto rendimiento y, además, disminuyen la cantidad de lodos residuales.

El sistema SBR con biomasa suspendida puede remover relativamente mejor los contaminantes orgánicos en términos de eliminación de carbono (C), en comparación 
con el rendimiento de los sistemas convencionales de crecimiento suspendido. Así, por ejemplo, se reportan eliminaciones de más del $90 \%$ en Demanda Bioquímica de Oxígeno (DBO), mientras que en los procesos convencionales se encuentran entre 60 y $75 \%$.

La inclusión de un medio de soporte en la cámara de reacción puede favorecer el crecimiento microbiano, debido a la retención de la biomasa nitrificante, que disminuye la retención del lodo y el tiempo de retención hidráulica (TRH) del proceso. En consecuencia, los sistemas que trabajan con crecimiento adherido con frecuencia se emplean en procesos de lodos activados modificados para mejorar la remoción de compuestos orgánicos y promover las tasas de nitrificación. Por otro lado, esta condición mejora la sedimentabilidad del lodo.

Es de gran importancia que se continúe con nuevas investigaciones en este campo, de tal forma que proporcionen el conocimiento necesario para determinar la posible combinación de las tecnologías de crecimiento de lechos microbianos adheridos (biofilm) y suspendidos, bajo secuencias anaerobias, aerobias y anóxicas en los reactores discontinuos secuenciales (SBR). Esto, con el fin de incrementar las eficiencias de remoción de materia orgánica y nutrientes de las aguas residuales.

\section{REFERENCIAS}

[1] Boaventura, K. M., Roqueiro, N., Coelho, M.A.Z. \& Araújo, O.Q.F. (2001). State observers for a biological wastewater nitrogen removal process in a sequential batch reactor. Bioresource Technology, 79(1), pp. 1-14.

[2] Carrera, J., Baeza, J., Vicent, T. \& Lafuente, J. (2003). Biological nitrogen removal of high-strength ammonium industrial wastewater with two-sludge system. Water Research, 37(17), pp. 4211-4221.

[3] Carrera, J., Vicent, T. \& Lafuente, J. (2004). Effect of influent COD/N ratio on biological nitrogen removal (BNR) from high-strength ammonium industrial wastewater. Process Biochemistry, 39(12), pp. 2035-2041.

[4] Fernandez M., F.J. (2006). Efectos de vertidos industriales sobre la eliminación biológica de nutrientes de aguas residuales urbanas (Tesis doctoral inédita). Universidad de Castilla la Mancha, Ciudad Real, España. p. 383.

[5] Akhbari, A., Zinatizadeh, A.A.L., Mohammadi, P., Irandoust, M. \& Mansouri, Y. (2011). Process modeling and analysis of biological nutrients removal in an integrated RBC-AS system using response surface methodology. Chemical Engineering Journal, 168(1), pp. 269-279.

[6] Li, J., Xing, X.H., \& Wang, B.Z. (2003). Characteristics of phosphorus removal from wastewater by biofilm sequencing batch reactor (SBR). Biochemical Engineering Journal, 16(3), pp. 279-285. 
[7] Rahimi, Y., Torabian, A., Mehrdadi, N. \& Shahmoradi, B. (2011). Simultaneous nitrification-denitrification and phosphorus removal in a fixed bed sequencing batch reactor (FBSBR). Journal of Hazardous Materials, 185(23), pp. 852-857.

[8] Di laconi, C., Lopez, A., Ramadori, R., Di Pinto, A.C. \& Passino, R. (2002). Combined chemical and biological degradation of tannery wastewater by a periodic submerged filter (SBBR). Water Research, 36(9), pp. 2205-2214.

[9] Ge, S., Peng, Y., Wang, S., Guo, J., Ma, B., Zhang, L. \& Cao, X. (2010). Enhanced nutrient removal in a modified step feed process treating municipal wastewater with different inflow distribution ratios and nutrient ratios. Bioresource Technology, 101(23), pp. 9012-9019.

[10] Mata-Alvarez, J., Obaja, D., Macé, S., Costa, J. \& Sans, C. (2003). Nitrification, denitrification and biological phosphorus removal in piggery wastewater using a sequencing batch reactor. Bioresource Technology, 87(1), pp. 103-111.

[11] Akın, B. S. \& Ugurlu, A. (2005). Monitoring and control of biological nutrient removal in a Sequencing Batch Reactor. Process Biochemistry, 40(8), pp. 2873-2878.

[12] Dautan, R., Pérez, M.L., Contreras, A., Marzana, A. \& Rincones, B. (noviembre, 1988). Diseño y construcción de un reactor discontinuo secuencial para remoción de DBO. Ponencia presentada en el XXVI Congreso Interamericano de Ingeniería Sanitaria y Ambiental, Lima, Perú.

[13] Cárdenas, C., Perruolo, T., Tarre, Y., Flores, K., Trujillo, A., Saules, L., Araujo, I. \& Yabroudi, S. (2006).
Remoción de nutrientes en un reactor discontinuo secuencial. Interciencia, 31(11), pp. 787-793.

[14] Tchobanoglous, G. \& Crites, R. (2003). Tratamiento de Aguas Residuales en pequeñas poblaciones. Bogotá, Colombia: Mc Graw Hill.

[15] Casellas, M., Dagot, C. \& Baudu, M. (2006). Set up and assessment of a control strategy in a SBR in order to enhance nitrogen and phosphorus removal. Process Biochemistry, 41(9), pp. 1994-2001.

[16] Arun, V, Mino. T. \& Mastsuo, T. (1988). Biological mechanism of acetate uptake mediated by carbohydrate consumption in excess phosphorus removal systems. Water Research, 22 (5), pp. 565-570.

[17] Mohan, S.V., Rao, N.C., Prasad, K.K., Madhavi, B.T.V. \& Sharma, P.N. (2005). Treatment of complex chemical wastewater in a sequencing batch reactor (SBR) with an aerobic suspended growth configuration. Process Biochemistry, 40(5), pp. 1501-1508.

[18] Zhang, Z., Zhu, J., King, J. \& Li, W. (2006). A two-step fed SBR for treating swine manure. Process Biochemistry, 41(4), pp. 892-900.

[19] Uygur, A. (2006). Specific nutrient removal rates in saline wastewater treatment using sequencing batch reactor. Process Biochemistry, 41(1), pp. 61-66.

[20] Mohan, S.V., Rao, N.C., \& Sarma, P.N. (2007). Low-biodegradable composite chemical wastewater treatment by biofilm configured sequencing batch reactor (SBBR). Journal of Hazardous Materials, 144(1-2), pp. 108-117. 
[21] Pavšelj, N., Hvala, N., Kocijan, J., Roš, M., Šubelj, M., Mušič, G. \& Strmčnik, S. (2001). Experimental design of an optimal phase duration control strategy used in batch biological wastewater treatment. ISA Transactions, 40(1), pp. 41-56.

[22] Kishida, N., Kim, J.H., Chen, M., Sasaki, H. \& Sudo, R. (2003). Effectiveness of oxidation-reduction potential and $\mathrm{pH}$ as monitoring and control parameters for nitrogen removal in swine wastewater treatment by sequencing batch reactors. Journal of Bioscience and Bioengineering, 96(3), pp. 285-290.

[23] Andreottola, G, Foladori, P, Ragazzi, M. (2000). On-line control of a SBR system for nitrogen removal from industrial wastewater. Proceedings of the second international symposium on SBR technology, pp. 154-62.

[24] Castelló E., Barcia R., Passeggi M., López. \& Borzaccioni L. (noviembre, 2006). Diseño, arranque y operación de reactor en secuencia batch a escala piloto para tratamiento de aguas. Ponencia presentada en el XXX Congreso Interamericano de Ingeniería Sanitaria y Ambiental. Punta del Este, Uruguay.

[25] Fu, Z., Yang, F., An, Y. \& Xue, Y. (2009). Simultaneous nitrification and denitrification coupled with phosphorus removal in an modified anoxic/oxic-membrane bioreactor (A/O-MBR). Biochemical Engineering Journal, 43(2), pp. 191-196.

[26] Wilderer, P.A. \& McSwain, B.S. (2004). The SBR and its biofilm application potentials. Water Science and Technology, 50(10), pp. 1-10.

[27] Rittmann, B.E. \& Langeland, W.E. (1985). Simultaneous denitrification with nitrification in single-channel oxidation ditches. Journal of Water Pollution Control Fed. 57, pp. 300-308.

[28] Rodgers, M. \& Zhan, X.-M. (2004). Biological nitrogen removal using a vertically moving biofilm system. Bioresource Technology, 93(3), pp. 313-319.

[29] Rittman, B.E. \& McCarty P.L. (Eds.). (2002) Environmental Biotechnology: Principles and Applications. Beijing, China: Tsinghua University Press, pp. 209-260.

[30] Broch, P.S. (2008). Operación y control de un proceso SBR para la remoción biológica mejorada de nutrientes de un agua residual. Universidad de Girona.

[31] Puig S., Vives M.T., Corominas L., Balaguer M.D. \& Colprim, J. (2004). Wastewater nitrogen removal in SBRs, applying a step-feed strategy: from lab-scale to pilot-plant operation. Water Science Technology, 50(10), pp. 89-96.

[32] Metcalf \& Eddy. (2003). Wastewater engineering: treatment and reuse. Nueva York, NY, EE.UU.: Mc Graw Hill.

[33] Singh, M. \& Srivastava, R.K. (2011). Sequencing batch reactor technology for biological wastewater treatment. Asia Pacific Journal of Chemical Engineering, 6(1), pp. 3-13.

[34] Stricker, A. \& Béland, M. (2006). Sequencing batch reactor versus continuos flow process for pilot plant research on activated sludge. Water Environment Foundation, pp. 7046-7056.

[35] Romero Rojas, J.A. (2004). Tratamiento de aguas residuales. Bogotá, Colombia: Escuela Colombiana de Ingeniería. p. 1197.

[36] Yang, S., Yang, F., Fu, Z., Wang, T. \& Lei, R. (2010). Simultaneous nitrogen 
and phosphorus removal by a novel sequencing batch moving bed membrane bioreactor for wastewater treatment. Journal of Hazardous Materials, 175/13), pp. 551-557.

[37] Wang, L.K., Shammas, N.K., \& Hung, Y.T. (2009). Advanced Biological Treatment Processes. New York. Humana Pre.

[38] Atlas R. \& Barttha, R. (2002). Ecología microbiana y Microbiología ambiental. Madrid, España: Pearson Educación S.A.

[39] Focht, D. (1982). Denitrification. En Burns R. \& Slater J. (Eds.). Experimental Microbial Ecology. Oxford, Inglaterra: Blackwell Science.

[40] Mahvi, A.H. (2008). Sequencing batch reactor: a promising technology in wastewater treatment. Journal of Environmental Health, Science Engineering, 5(2), pp. 79-90.

[41] Scaler, M. I. \& Mujeriego, R. (2001). Eliminación Biológica de Nutrientes (Nitrógeno y Fósforo) Mediante un Proceso Discontinuo de Fangos Activados. Ingeniería Del Agua, 8, pp. 67-78.

[42] Tchobanoglous, G., Burton, F.L. \& Stensel, H.D. (2003). Wastewater Engineering: Treatment and Reuse. Nueva York, NY, EE.UU: McGraw Hill.

[43] Cui, Y., Wang, S. \& Li, J. (2009). On-line Monitoring for Phosphorus Removal Process and Bacterial Community in Sequencing Batch Reactor. Chinese Journal of Chemical Engineering, 17 (3), pp. 484- 92.

[44] Carvalho, G., Lemos, P.C., Oehmen, A., \& Reis, M.A.M. (2007). Denitrifying phosphorus removal: Linking the process performance with the microbial community structure. Water Research, 41(19), pp. 4383-4396.

[45] Whang, L.M., Filipe, C.D.M., \& Park, J.K. (2007). Model-based evaluation of competition between polyphosphateand glycogen-accumulating organisms. Water Research, 41(6), pp. 1312-1324.

[46] Oehmen, A., Saunders, A.M., Vives, M.T., Yuan, Z. \& Keller, J. (2006). Competition between polyphosphate and glycogen accumulating organisms in enhanced biological phosphorus removal systems with acetate and propionate as carbon sources. Journal of Biotechnology, 123(1), pp. 22-32.

[47] Lee, D.S., Jeon, C.O. \& Park, J.M. (2001). Biological nitrogen removal with enhanced phosphate uptake in a sequencing batch reactor using single sludge system. Water Research, 35(16), pp. 3968-3976.

[48] Al-Rekabi, W.S., Qiang, H. \& Qiang, W.W. (2007). Review on Sequencing Batch Reactors. Pakistan Journal of Nutrition, 6(1), pp. 11-19.

[49] Zeng, R.J. \& Yuan, Z. (2004). Improved understanding of the interactions and complexities of biological nitrogen and phosphorus removal processes. Bioengineering, 3, pp. 265-272.

[50] Wang, F., Lu, S., Wei, Y. \& Ji, M. (2009). Characteristics of aerobic granule and nitrogen and phosphorus removal in a SBR. Journal of Hazardous Materials, 164(2-3), pp. 1223-1227.

[51] Miao, L., Wang, K., Wang, S., Zhu, R., Li, B., Peng, Y. \& Weng, D. (2014). Advanced nitrogen removal from landfill leachate 
using real-time controlled three-stage sequence batch reactor (SBR) system. Bioresource Technology, 159, pp. 258-265.

[52] Wei, Y., Ji, M., Li, R. \& Qin, F. (2012). Organic and nitrogen removal from landfill leachate in aerboci granular sludge sequencing batch reactors. Waste Management, 32, pp. 448-455.

[53] Uygur, A \& Kargi, F. (2004). Biological nutrient removal from pre-treated landfill leachate in a sequencing batch reactor. Journal of Environmental Management, 71, pp. 9-14.

[54] Morling, S. (2010). Nitrogen removal and heavy metals in leachate treatment using SBR techonology. Journal of Hazardous Materials, 174, pp. 679-686.

[55] Neczaj, E., Kacprzak, M., Kamizela, T., Lach, J. \& Okoniewska, E. (2008). Sequencing Batch reactor system for the co-treatment of landfill leachate and dairy wastewater. Desalination, 222, pp. 404-409.

[56] Laitinen, N., Luonsi, A. \& Vilen, J. (2006). Landfill leachate treatment with sequencing batch reactor and membrane bioreactor. Desalination, 191, pp. 86-91.

[57] Huang, X., Gao, D., Peng, S. \& Tao, Y. (2014). Effects of ferrous and manganese ions on anammox process in sequencing batch biofilm reactors. Journal of Environmental Sciences, 26, pp. 1034-1039.

[58] Pérez, M. L., Dauntan, R., Contreras, A. \& González, H. P. (2002). Remoción de nitrógeno y fósforo en aguas residuales utilizando un reactor discontinuo secuencial SBR. Presentado en el XXVIII Congreso Interamericano de Ingeniería Sanitaria y Ambiental. Cancún, México.
[59] Wang, D., Li, X., Yang, Q., Zeng, G., Liao, D. \& Zhang, J. (2008). Biological phosphorus removal in sequencing batch reactor with single-stage oxic process. Bioresource Technology, 99(13), pp. 5466-5473.

[60] Wu, Ch. \& Jin, Y. (2011). Performance of nitrogen and phosphorus removal of municipal wastewater in sequencing batch biofilm reactor. Energy Procedia, 11, pp. 4453-4457.

[61] Feng, Ch., Ding, D., Jin, Y., Hao, Ch., Zhao, Y. \& Suemura, T. (2011). Domestic sewage treatment in a sequencing batch biofilm reactor (SBBR) with an intelligent controlling system. Desalination, 276, pp. 260-265.

[62] Sirianuntapiboon, S., Jeeyachok, N. \& Larplai, R. (2005). Sequencing batch reactor biofilm system for treatment of milk industry wastewater. Journal of Environmental Management, 76(2), pp. 177-183.

[63] Kim, D., Jung, N. \& Park, Y. (2008). Characteristics of nitrogen and phosphorus removal in SBR and SBBR with different ammonium loading rates. Korean J. Chem. Eng., 25(4), pp. 793-800.

[64] Sirianuntapiboon, S. \& Sansak, J. (2008). Treatability studies with granular activated carbon and sequencing batch reactor system for textile wastewater containing direct dyes. Journal of Hazardous Materials, 159, pp. 404-411.

[65] Malakahmad, A., Hasani, A., Eisakhani, M. \& Isa, M. (2011). Sequencing batch reactor for the removal of $\mathrm{Hg}$ and $\mathrm{Cd}$ from synthetic petrochemical factory wastewater. Journal of Hazardous Materials, 191, pp. 118-125. 
[66] Lin, S. \& Jiang, Ch. (2003). Fenton oxidation and sequencing batch reactor treatments of high-strength semiconductor wastewater. Desalination, 154, pp. 107-116.

[67] Van Den Hende, S., Carré, E., Cocaud, E., Beelen, V., Boon, N .\& Vervaeren, H. (2014). Treatment of industrial wastewaters by microalgal bacterial flocs in sequencing batch reactors. Bioresource Technology, 161, pp. 245-254.

[68] Marañón, E., Vázquez, I., Rodríguez, J., Castrillón, L., Fernández, Y. \& López, H. (2008). Treatment of coke wastewater in a sequential batch reactor at pilot plant scale. Bioresource Techonolgy, 99, pp. 4192-4198.

[69] Bural, C., Demirer, G., Kantoglu, O. \& Dilek, F. (2010). Treatment of opium alkaloid containing wastewater in sequencing batch reactor-Effect of gamma irradiation. Radiation Physics and Chemistry, 79, pp. 519-526.

[70] Daverey, A., Su, S., Huang, Y., Chen, S., Sung, S. \& Lin, J. (2013). Partial nitrification and anammox process: A method for high strength optoelectronic industrial wastewater treatment. Water Research, 47, pp. 2929-2937.

[71] Lan, Ch., Kumar, M., Wang, Ch.\& Lin, J. (2011). Development of simultaneous partial nitrification process in a sequential batch reactor. Bioresource Technology, 102, pp. 5514-5519. 